\title{
Neuropsychological correlates underlying verbal fluency deficits in schizophrenia: The role of attention and executive function
}

\author{
Anna Rosenkranz - Tilo Kircher $^{2} \cdot$ Arne Nagels $^{3}$ \\ 'University of Cologne, Faculty of Human Sciences, Department of Special Education and \\ Rehabilitation, ${ }^{2}$ Philipps University Marburg, Department of Psychiatry and Psychotherapy \\ ${ }^{3}$ Johannes Gutenberg University Mainz, Department of English and Linguistics, General Linguistics
}

https://doi.org/10.17234/9789531758314.04

\begin{abstract}
Verbal fluency deficits are commonly observed in patients with schizophrenia. The role of executive functions is still a topic of critical debate, although other neuropsychological domains, e.g. attention, working memory and verbal intelligence, may also influence verbal fluency task performance. Furthermore, some studies report semantic fluency dysfunctions suggesting particular semantic access deficits, while others report regular patterns of verbal fluency task performance, with better performance in semantic fluency as compared to lexical fluency, suggesting general retrieval difficulties. In addition, formal thought disorder symptomatology is often associated with reduced verbal fluency performance. To further address these issues of verbal fluency deficits in schizophrenia, patients with schizophrenia $(n=50)$ as well as a healthy control group $(n=36)$ were recruited and tested according to the aforementioned neuropsychological parameters and both semantic and lexical fluency. Results indicate that executive dysfunctions are associated with verbal fluency performance particularly in the lexical fluency domain. Furthermore, a strong relationship was found between sustained-attention deficits and both semantic and lexical fluency, indicating that verbal fluency deficits in general are mainly driven by attention dysfunctions rather than aberrations in the executive domain. Results showed that patients with schizophrenia did not perform differently for letter vs. semantic fluency. Furthermore, controlling for attention, the results suggest that formal thought disorder symptomatology is unrelated to performance on verbal fluency. Taken together, impaired attention drives the decreased performance in verbal fluency tasks in patients with schizophrenia.
\end{abstract}

Key words: semantic fluency, lexical fluency, schizophrenia, neuropsychology, executive function, attention, language, alogia, formal thought disorder 


\section{Theoretical background}

Deficits in verbal fluency performance are commonly observed in patients with schizophrenia (Bokat \& Goldberg, 2003; Henry \& Crawford, 2005). The two most common verbal fluency tasks are the semantic (or category) fluency task, in which participants are asked to generate as many words as possible belonging to a specific semantic category, and the lexical (or letter) fluency task, in which participants are asked to generate as many words as possible belonging to a specific initial letter or initial phoneme. Literal repetitions are not allowed in either task, and word-stem repetitions and proper names are off limits in the lexical fluency task. The given time window, which is between one and five minutes, varies among the different studies (Mitrushina, Boone, Razani \& D'Elia, 2005; Raboutet, Sauzéon, Corsini, Rodrigues, Langevin \& N'Kaoua, 2010). From a neuropsychological perspective, verbal fluency "has been commonly viewed as a component of executive function" (Mitrushina et al., 2005:202). However, the particular role of executive functions - in this context it is often referred to as "dysexecutive syndrome" (Thai, Andreassen \& Bliksted, 2019) - on verbal fluency task performance is still a topic of critical debate. Other cognitive domains, such as attention, but also working memory and verbal intelligence, may also influence task performance. On a linguistic level, verbal fluency involves both efficient word retrieval processes and intact access to semantic or orthographic representations (Shao, Janse, Visser \& Meyer, 2014; Stielow, 2017). With regard to patients with schizophrenia, semantic fluency dysfunctions were reported (Bokat \& Goldberg, 2003; Henry \& Crawford, 2005), suggesting particular semantic access deficits. In this context, category switching and clustering performance was found to be aberrant (Allen, Liddle \& Frith, 1993; Elvevåg, Fisher, Gurd \& Goldberg, 2002), supporting the view that semantic organization within the lexicon is impaired. On the other hand, some patient studies also report regular patterns of verbal fluency task performance, with better performance in semantic fluency than in lexical fluency (Barrera, McKenna \& Berrios, 2005; Docherty, Berenbaum \& Kerns, 2011; Doughty \& Done, 2009), suggesting general retrieval difficulties. These differences may be driven by the presented stimuli per se (large [e.g. 'animals'] vs. small [e.g. 'professions'] semantic or lexical fields) as well as by the given time window. Other influencing factors are associated with certain search strategies such as mental imagery (e.g. walking through a zoo) and the presence of formal thought disorder symptomatology. Formal thought disorder is used as a collective term to describe dysfunctions in the formal production or perception of the patients' language, e.g. perseveration or derailment (Kircher et al., 2014; Nagels et al., 2016). Traditionally, a distinction has been made between positive and negative formal thought disorder dimensions. Positive formal thought disorder is associated with disorganized 
language output, whereas negative formal thought disorder - often referred to as alogia or poverty of speech - is associated with reduced speech and language output. Verbal fluency deficits have been found in both negative (Joyce, Collinson \& Crichton, 1996) and positive formal thought disorder (Goldberg, Aloia, Gourovitch, Missar, Pickar \& Weinberger, 1998). Docherty et al. (2011) showed that negative formal thought disorder was related with poor controlled retrieval on semantic fluency whereas positive formal thought disorder was related with poor context processing on letter fluency. In contradiction, Egeland, Holmen, Bang-Kittilsen, Bigseth and Engh (2018) reported that positive symptoms were positively related to semantic fluency, suggesting "that this may be due to more efficient spread of associations" (p. 38).

Taken together, many domains seem to affect verbal fluency performance in patients with schizophrenia. The purpose of the current investigation is to identify neuropsychological correlates underlying verbal fluency deficits in schizophrenia and to examine semantic and lexical fluency performance in patients with schizophrenia compared to healthy controls. In addition, it investigates whether positive and negative formal thought disorders influence verbal fluency performance.

\section{Methods}

\subsection{Participants}

This study included 50 in- and outpatients diagnosed with schizophrenia (F20.x; 12 females and 38 males; mean age 35.5 years, SD = 11.2) (ICD-10) aged between 19 and 60 years. In- and out-patients were recruited and interviewed at the Department of Psychiatry and Psychotherapy, Philipps University Marburg.

A group of 36 age-matched healthy participants (22 females and 14 males; mean age 36.6 years, $\mathrm{SD}=11.3$ ) aged between 18 and 59 years was recruited by means of newspaper advertisements.

\subsection{Procedure}

\section{Clinical Assessment Procedure}

Positive formal thought disorder was measured using the Scale for the Assessment of Positive Symptoms (SAPS) (Andreasen, 1984a). The dependent measure was the subscale score for positive formal thought disorder (subscale IV).

Negative formal thought disorder was measured using the Scale for the Assessment of Negative Symptoms (SANS) (Andreasen, 1984b). The dependent measure was the subscale score for alogia (subscale II). 


\section{Verbal Fluency Assessment Procedure}

Verbal fluency performance was assessed using semantic and lexical fluency tasks (Aschenbrenner, Lange \& Tucha, 2000). For the semantic fluency task, the participants were instructed to generate as many words as possible beginning with the semantic category animals within one minute. In the lexical fluency task, the participants were instructed to generate as many words as possible beginning with the letter $p$ within one minute. The dependent measure for both verbal fluency tasks was the number of correctly generated words, excluding repetitions, and rule-breakings.

\section{Neuropsychological Assessment Procedure}

The participants' executive functioning abilities were assessed using the Trail Making Test (TMT), parts A and B (Reitan, 1958). In task A, the participants were instructed to connect numbers in ascending order (e.g. 1-2-3). In task B, the participants were instructed to connect numbers and letters alternately (e.g. 1-A-2-B). The participants were asked to finish task A and task B as quickly as possible. The dependent measure was the difference in seconds between the duration of parts $\mathrm{A}$ and $\mathrm{B}$.

The D2 test of attention (Brickenkamp, 2002), a paper-and-pencil test, was used to assess the participants' sustained and selective attention processes. The participants were asked to mark any letter $d$ with two apostrophes (d") and neglect distractors similar to the target stimulus (e.g. d'). The dependent measure was the raw score of the concentration performance parameter.

The participants' working memory was assessed using the digit span backwards task taken from the Wechsler Memory Scale (WMS) (Wechsler, 1987). In the backwards task, the participants were instructed to memorize and reproduce a sequence of digits in the reverse order. The dependent measure was the raw score of the digit span backwards task.

The MWT-B (Lehrl, 1989), a multiple-choice vocabulary test, was used to assess the participants' verbal intelligence. The participants were asked to identify the correct written word from a list including distractors similar to the target word. The dependent measure was the raw score of all 37 correctly identified words.

All participants completed all tests with the following exceptions. One patient with schizophrenia failed to complete the TMT, and one patient with schizophrenia failed to complete the digit span backwards task. 


\subsection{Data Analysis}

The Statistical Package for Social Sciences (SPSS 25) software package was used to perform all analyses. Dependent measures from the TMT, the D2 test, the digit span backwards task, and the MWT-B were subjected to unpaired t-tests. A Spearman's correlation was computed between (a) the number of correctly generated words in both semantic and lexical fluency tasks, and (b) the dependent measures from the TMT, the D2 test, the digit span backwards task, and the MWT-B as well as for the SAPS (subscale IV) and SANS (subscale II) subscale scores. Whenever neuropsychological test performance was correlated with the SAPS and SANS subscale scores, the effect of neuropsychological test performance on other correlations was examined by performing partial correlation coefficients. Semantic and lexical fluency data were analyzed with two-factor analyses of variance (the factors group and condition) with repeated measures on the second factor.

\section{Results}

\subsection{Neuropsychological correlates underlying verbal fluency deficits}

As shown in Table 1, the dependent measures of all neuropsychological assessments (the difference score in seconds for the TMT, the raw score of the concentration performance parameter for the D2 test, the raw score of the digit span backwards task, and the raw score of all correctly identified words on the MWT-B) revealed statistically significant differences, indicating that the group of patients performed significantly worse in all neuropsychological assessments in comparison to the control group.

Table 1. Results of the neuropsychological assessments for patients with schizophrenia (PwS) and healthy controls $(\mathrm{HC})$.

\begin{tabular}{|l|r|r|r|}
\hline & \multicolumn{1}{|c|}{ PwS } & \multicolumn{1}{|c|}{ HC } & \\
\hline TMT & $60.9(34.3)$ & $29.0(15.3)$ & $\begin{array}{r}\mathrm{t}(70.51)=-5.78 ; \\
\mathrm{p}<.001\end{array}$ \\
\hline D2 test & $111.1(36.8)$ & $166.3(38.0)$ & $\mathrm{t}(84)=6.77 ; \mathrm{p}<.001$ \\
\hline Digit span backwards & $5.5(1.6)$ & $7.8(2.0)$ & $\mathrm{t}(83)=5.76 ; \mathrm{p}<.001$ \\
\hline MWT-B & $27.5(5.6)$ & $31.7(2.8)$ & $\begin{array}{r}\mathrm{t}(75.96)=4.65 ; \\
\mathrm{p}<.001\end{array}$ \\
\hline
\end{tabular}

Note. Standard deviation is given in brackets. Additionally, the results of unpaired t-tests are given in the right column. For the TMT, higher difference scores indicate poorer performance.

No evidence for a relationship between executive functions $\left(\mathrm{r}_{\mathrm{s}}=-.253, \mathrm{p}=.079\right)$, working memory task performance $\left(\mathrm{r}_{\mathrm{s}}=.106, \mathrm{p}=.468\right)$ and verbal intelligence $\left(\mathrm{r}_{\mathrm{s}}=.250, \mathrm{p}=.080\right)$ and semantic fluency task performance was observed. Per- 
formance on executive functions $\left(\mathrm{r}_{\mathrm{s}}=-.322, \mathrm{p}=.024\right)$, working memory task performance $\left(r_{s}=.343, p=.016\right)$ and verbal intelligence $\left(r_{s}=.287, p=.043\right)$ were significantly correlated with the number of correctly generated words only in the lexical fluency task. A strong relationship was found between sustained-attention deficits and both dimensions, semantic $\left(\mathrm{r}_{\mathrm{s}}=.443, \mathrm{p}=.001\right)$ and lexical fluency $\left(\mathrm{r}_{\mathrm{s}}=.403, \mathrm{p}=.004\right)$.

\subsection{Verbal fluency performance in patients with schizophrenia}

Regarding the number of correctly generated words (see Figure 1), an ANOVA revealed a main effect of task $(F[1,84]=335.56, p<.001)$ and group $(F[1,84]=$ $16.28, p<.001$ ), showing that the group of controls generated significantly more correct words than the group of patients and that both groups generated significantly more correct words in semantic fluency than in lexical fluency. There was no interaction effect between task and group $(F[1,84]=1.96, p=.166)$.

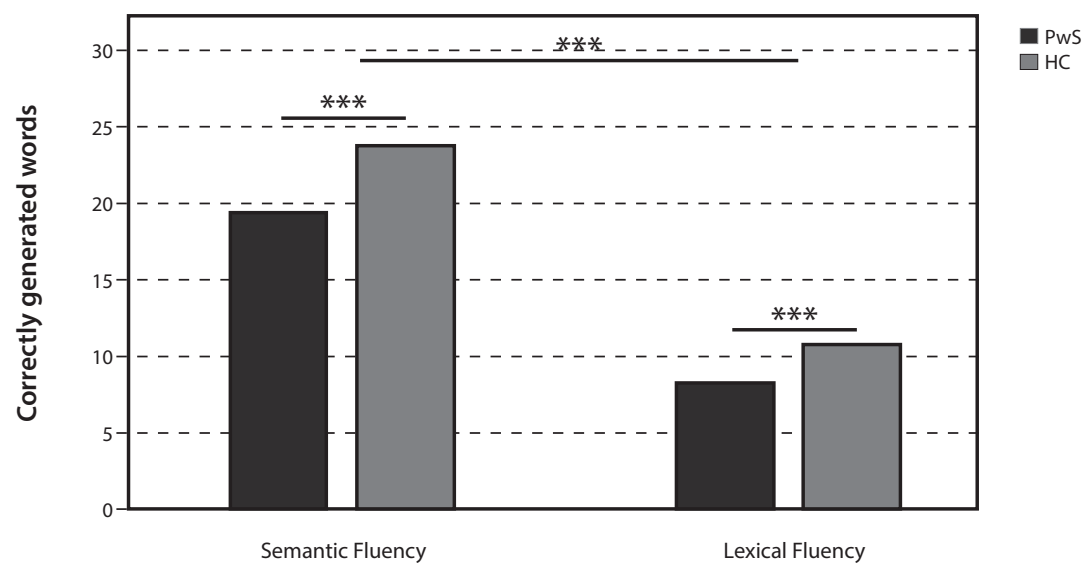

Figure 1. Graphs showing performance on both semantic and lexical fluency (mean number of correctly generated words) of the group of patients with schizophrenia (PwS) and the group of healthy controls (HC).

\subsection{Influence of formal thought disorder on verbal fluency}

The SAPS subscale score for positive formal thought disorder was not significantly correlated with the number of correctly generated words neither in the semantic $\left(\mathrm{r}_{\mathrm{s}}=.079, \mathrm{p}=.584\right)$ nor in the lexical fluency task $\left(\mathrm{r}_{\mathrm{s}}=.102, \mathrm{p}=.482\right)$. The SANS subscale score for negative formal thought disorder was significantly correlated with the number of correctly generated words in the semantic fluency task $\left(\mathrm{r}_{\mathrm{s}}=-.420, \mathrm{p}=.002\right)$ but not in the lexical fluency task $\left(\mathrm{r}_{\mathrm{s}}=-.251, \mathrm{p}=.079\right)$.

Positive formal thought disorder was not significantly correlated with selective and sustained attention $\left(r_{s}=-.198, p=.168\right)$ whereas negative formal thought disorder was significantly correlated with selective and sustained at- 
tention $\left(\mathrm{r}_{\mathrm{s}}=-.352, \mathrm{p}=.012\right)$. No significant correlations between positive and negative formal thought disorder and executive functions, working memory and verbal intelligence were found. A partial correlation was then computed between subscale scores for negative formal thought disorder and performance on semantic $(\mathrm{r}=-.152, \mathrm{p}=.296)$ and lexical fluency $(\mathrm{r}=-.145, \mathrm{p}=.321)$, controlling for attention.

\section{Discussion}

The aim of the present study was to identify neuropsychological correlates underlying verbal fluency deficits in schizophrenia. The results indicate that executive dysfunctions are associated with verbal fluency performance particularly in the lexical fluency domain. Furthermore, a strong relationship was found between sustained-attention deficits and both semantic and lexical fluency, indicating that verbal fluency deficits in general are mainly driven by sustained-attention dysfunctions rather than aberrations in the executive domains. Furthermore, the current study showed that patients with schizophrenia did not perform differently comparing the relation of letter and semantic fluency. Previous research had reported distinctly different findings, with studies reporting disproportionate impairment on semantic fluency tasks and arguing for semantic rather than executive difficulties (Bokat \& Goldberg, 2003; Henry \& Crawford, 2005) and others reporting a normal pattern of verbal fluency task performance, with better performance on semantic fluency than on lexical fluency, arguing for general retrieval difficulties (Barrera et al., 2005; Docherty et al., 2011; Doughty \& Done, 2009). These reported differences may be driven by the variety of the presented stimuli as well as the presence of formal thought disorder symptomatology. Previous studies found verbal fluency deficits in both negative (Joyce et al., 1996) and positive formal thought disorder (Goldberg et al., 1998), as well as contradicting results, showing that positive symptoms were positively related to semantic fluency (Egeland et al., 2018). Correlation analysis in the current study showed that only negative formal thought disorder - often referred to as alogia or poverty of speech - was adversely related with performance on semantic fluency. However, controlling for attention, the results suggest that negative formal thought disorder are unrelated to performance on verbal fluency. In line with aforementioned results, impaired attention drives the decreased performance in verbal fluency tasks in patients with schizophrenia. However, it should be noted that it is challenging to determine tasks that properly test complex cognitive processes. This issue requires future research using additional assessments before definitive conclusions can be made. Additionally, traditional diagnostic procedures of verbal fluency output analyze responses with respect to the number of correctly generated words only. To identify the underlying problem of limited output in verbal 
fluency in schizophrenia, it might be beneficial to use additional procedures to analyze word generation performance such as clustering and switching component scores (Bozikas, Kosmidis \& Karavatos, 2005; Egeland et al., 2018; Robert et al., 1998) as well as temporal parameters (Docherty et al., 2011).

\section{Limitations}

Although this study provided detailed descriptions of a variety of factors, the neuropsychological deficits found in schizophrenia vary depending on additional factors, such as on the effect of age, the effect of the duration and phase of the illness, and the effects of medication. Additionally, there are a variety of factors affecting cognition even in healthy control participants, such as the effect

of physical activity as well as the effect of possible use of alcohol or drugs, that were not analyzed in the participants.

\section{Acknowledgements}

The authors would like to thank Paul Fährmann, Maria Gassmann, Sayed Ghazi, Christian Schales, and Lena Turner.

Anna Rosenkranz is supported by the German Ministry for Education and Research (BMBF, reference number: 01UL1812X).

\section{References}

Allen, H. A., Liddle, P. F., \& Frith, C. D. (1993). Negative features, retrieval processes and verbal fluency in schizophrenia. The British Journal of Psychiatry: The Journal of Mental Science, 163, 769-775.

Andreasen, N. (1984a). The Scale for the Assessment of Negative Symptoms (SANS). Iowa City: University of Iowa.

Andreasen, N. (1984b). The Scale for the Assessment of Positive Symptoms (SAPS). Iowa City: University of Iowa.

Aschenbrenner, S., Lange, K. W., \& Tucha, O. (2000). RWT: Regensburger Wortflüssigkeits-Test. Göttingen: Hogrefe.

Barrera, A., McKenna, P. J., \& Berrios, G. E. (2005). Formal thought disorder in schizophrenia: an executive or a semantic deficit? Psychological Medicine, 35, 121-132.

Bokat, C. E., \& Goldberg, T. E. (2003). Letter and category fluency in schizophrenic patients: A meta-analysis. Schizophrenia Research, 64, 73-78.

Bozikas, V. P., Kosmidis, M. H., \& Karavatos, A. (2005). Disproportionate impairment in semantic verbal fluency in schizophrenia: Differential deficit in clustering. Schizophrenia research, 74, 51-59.

Brickenkamp, R. (2002). Test D2 Aufmerksamkeits-Belastungs-Test. Göttingen: Hogrefe. 
Docherty, A. R., Berenbaum, H., \& Kerns, J. G. (2011). Alogia and formal thought disorder: Differential patterns of verbal fluency task performance. Journal of Psychiatric Research, 45, 1352-1357.

Doughty, O. J., \& Done, D. J. (2009) Is semantic memory impaired in schizophrenia? A systematic review and meta-analysis of 91 studies. Cognitive Neuropsychiatry, 14(6), 473-509.

Egeland, J., Holmen, T. L., Bang-Kittilsen, G., Bigseth, T. T., \& Engh, J. A. (2018). Category fluency in schizophrenia: Opposing effects of negative and positive symptoms? Cognitive Neuropsychiatry, 23, 28-42.

Elvevåg, B., Fisher, J. E., Gurd, J. M., \& Goldberg, T. E. (2002). Semantic clustering in verbal fluency: Schizophrenic patients versus control participants. Psychological Medicine, 32(5), 909-917.

Goldberg, T.E., Aloia, M. S., Gourovitch, M. L., Missar, D., Pickar, D., \& Weinberger, D. R. (1998). Cognitive substrates of thought disorder, I: The semantic system. The American Journal of Psychiatry, 155(12), 1671-1676.

Henry J. D., \& Crawford, J. R. (2005). A meta-analytic review of verbal fluency deficits in schizophrenia relative to other neurocognitive deficits. Cognitive Neuropsychiatry, 10(1), 1-33.

Joyce, E. M., Collinson, S. L., \& Crichton, P. (1996). Verbal fluency in schizophrenia: relationship with executive function, semantic memory and clinical alogia. Psychological Medicine, 26(1), 39-49.

Kircher, T., Krug, A., Stratmann, M., Ghazi, S., Schales, C., Frauenheim, M., Turner, L., Fährmann, P., Hornig, T., Katzev, M., Grosvald, M., Müller-Isberner, R., \& Nagels, A. (2014). A rating scale for the assessment of objective and subjective formal Thought and Language Disorder (TALD). Schizophrenia Research, 160, 216-221.

Lehrl, S. (1989). Mehrfachwahl-Wortschatz-Intelligenztest: MWT-B. Erlangen: Perimed.

Mitrushina, M., Boone, K. B., Razani, J., \& D’Elia, L. F. (2005). Handbook of normative data for neuropsychological assessment. 2nd edition. New York: Oxford University Press.

Nagels, A., Fährmann, P., Stratmann, M., Ghazi, S., Schales, C., Frauenheim, M., Turner, L., Hornig, T., Katzev, M., Müller-Isberner, R., Grosvald, M., Krug, A., \& Kircher T. (2016). Distinct Neuropsychological Correlates in Positive and Negative Formal Thought Disorder Syndromes: The Thought and Language Disorder Scale in Endogenous Psychoses. Neuropsychobiology, 73(3), 139-47.

Raboutet, C., Sauzéon, H., Corsini, M.-M., Rodrigues, J., Langevin, S., \& N’Kaoua, B. (2010). Performance on a semantic verbal fluency task across time: Dissociation between clustering, switching, and categorical exploitation processes. Journal of Clinical and Experimental Neuropsychology, 32(3), 268-280.

Reitan, R. (1958). The validity of the trail making test as an indicator of organic brain damage. Perceptual and Motor Skills, 8, 271-276.

Robert, P. H., Lafont, V., Medecin, I., Berthet, L., Thauby, S., Baudu, C., \& Darcourt, G. (1998). Clustering and switching strategies in verbal fluency tasks: Comparison between schizophrenics and healthy adults. Journal of the International Neuropsychological Society, 4, 539-546. 
Shao, Z., Janse, E., Visser, K., \& Meyer, A. S. (2014). What do verbal fluency tasks measure? Predictors of verbal fluency performance in older adults. Frontiers in Psycho$\log y, 5(772)$.

Stielow, A. (2017). The contribution of executive functions to performance in word retrieval tasks in aphasic patients and healthy controls - A psycholinguistic investigation. Doctoral dissertation, Ruhr-University Bochum.

Thai, M. L., Andreassen, A. K., \& Bliksted, V. (2019). A meta-analysis of executive dysfunction in patients with schizophrenia: Different degree of impairment in the ecological subdomains of the Behavioural Assessment of the Dysexecutive Syndrome. Psychiatry Research, 272, 230-236.

Wechsler, D. (1987). WMS-R: Wechsler Memory Scale-Revised: Manual. San Antonio: Harcourt Brace Jovanovich. 